\title{
Minimal and Maximal Extent of Band Ligation for Acute Variceal Bleeding during the First Endoscopic Session
}

\author{
Jang Han Jung ${ }^{1}$, Jung Hyun Jo², Sung Eun Kim³, Chang Seok Bang ${ }^{4}$, Seung In Seo ${ }^{5}$, Chan Hyuk Park ${ }^{6}$, and Se \\ Woo Park ${ }^{1}$ \\ ${ }^{1}$ Division of Gastroenterology, Department of Internal Medicine, Hallym University Dongtan Sacred Heart Hospital, Hallym University \\ College of Medicine, Hwaseong, ${ }^{2}$ Division of Gastroenterology, Department of Internal Medicine, Yonsei University College of \\ Medicine, Seoul, ${ }^{3}$ Division of Gastroenterology, Department of Internal Medicine, Hallym University Sacred Heart Hospital, Hallym \\ University College of Medicine, Anyang, ${ }^{4}$ Division of Gastroenterology, Department of Internal Medicine, Hallym University Chuncheon \\ Sacred Heart Hospital, Hallym University College of Medicine, Chuncheon, ${ }^{5}$ Division of Gastroenterology, Department of Internal \\ Medicine, Gangdong Sacred Heart Hospital, Seoul, and ${ }^{6}$ Department of Internal Medicine, Hanyang University Guri Hospital, Hanyang \\ University College of Medicine, Guri, Korea
}

\section{Article Info}

Received December 30, 2020

Revised March 15, 2021

Accepted March 15, 2021

Published online August 30, 2021

\section{Corresponding Author \\ Se Woo Park \\ ORCID https://orcid.org/0000-0003-1603-7468 \\ E-mail britnepak@outlook.com}

\section{Chan Hyuk Park}

ORCID https://orcid.org/0000-0003-3824-3481

E-mail yesable7@gmail.com

Jang Han Jung and Jung Hyun Jo contributed equally to this work as first authors.
Background/Aims: The appropriate number of band ligations during the first endoscopic session for acute variceal bleeding is debatable. We aimed to compare the technical aspects of endoscopic variceal ligation (EVL) in patients with variceal bleeding according to the number of bands placed per session.

Methods: We retrospectively reviewed multicenter data from patients who underwent EVL for acute variceal bleeding. Patients were classified into minimal EVL (targeting only the foci with active bleeding or stigmata of recent bleeding) and maximal EVL (targeting potential bleeding sources in addition to the aforementioned targets) groups. The primary endpoint was 5-day treatment failure. The secondary endpoints were 30-day rebleeding, 30-day mortality, and intraprocedural adverse events.

Results: Minimal EVL was associated with lower rates of hypoxia and shock during EVL than maximal EVL (hypoxia, $0.9 \%$ vs $2.9 \%$; shock, $1.3 \%$ vs $3.4 \%$ ). However, treatment failure was higher in the minimal EVL group than in the maximal EVL group (odds ratio, 1.60; $95 \%$ confidence interval, 1.06 to 2.41). Age $\geq 60$ years, Model for End-Stage Liver Disease score $\geq 15$, Child-Turcotte-Pugh classification C, presence of hepatocellular carcinoma, and systolic blood pressure $<90 \mathrm{~mm} \mathrm{Hg}$ at initial presentation were also associated with treatment failure. In contrast, 30-day rebleeding and 30-day mortality did not differ between the minimal and maximal EVL groups.

Conclusions: Given that minimal EVL was associated with a high risk of treatment failure, maximal EVL may be a better option for variceal bleeding. However, the minimal EVL strategy should be considered in select patients because it does not affect 30-day rebleeding and mortality. (Gut Liver 2022;16:101-110)

Key Words: Endoscopic hemostasis; Band ligation; Gastrointestinal hemorrhage; Esophageal and gastric varices; Liver cirrhosis

\section{INTRODUCTION}

Endoscopic variceal ligation (EVL) has been found to be helpful when treating variceal bleeding during the first endoscopic session; therefore, it is used to reduce mortality resulting from rebleeding. ${ }^{1,2}$ However, mortality among pa- tients undergoing EVL remains high, and the reported rebleeding rates range from approximately $10 \%$ to more than $50 \% .^{3-5}$ The recent American Association for the Study of Liver Diseases practice guidelines suggest that EVL sessions should be repeated at 1 - to 4 -week intervals until obliteration of varix. ${ }^{6}$ This obliteration usually requires two 
to four sessions according to data collected from different randomized clinical trials. ${ }^{7,8}$ Within the allowable safety range for patient tolerability, endoscopic control of ligation is undoubtedly the best treatment option. ${ }^{9}$ However, in clinical practice, debate remains regarding how many varices should be targeted for bleeding control and eradication during the first endoscopic session for acute variceal bleeding.

Gastroenterologists are sometimes hindered by the noncooperation of patients during endoscopic procedures, and technical failure or fatal adverse events can occur in extreme cases. ${ }^{10}$ Therefore, reducing the procedure time is the most important factor for patient safety. Some endoscopists in Korea have tried to minimize the total procedure time by targeting only the variceal focus with current bleeding or those at high risk for stigmata, indicating recent bleeding (such as pin-point ulceration or fibrin plug). In contrast, it has been suggested that the more ligation bands that are applied, the earlier the eradication of varices while the fewer bands are applied, the more rebleeding within 6 months from the index event of bleeding. ${ }^{11,12}$ However, a recent randomized trial demonstrated that the application of more than six bands during any EVL session is not closely related to needs for fewer additional EVL sessions for total eradication, prevention of rebleeding or recurrence, or reduction of early/intermediate mortality rates. ${ }^{12}$ This strategy was also associated with longer procedure times and higher rates of misfiring bands.

Nevertheless, there are no data or guidelines that directly compare the minimal (performing targeted band ligation for only current oozing or spurting type bleeding from varix or stigmata of recent bleeding) and maximal (performing as many band ligations as possible for potential bleeding and active variceal bleeding) extent of EVL for hemostasis of acute variceal bleeding during the first endoscopic session. Therefore, we designed a retrospective cohort study to compare treatment failure, rebleeding and mortality within 30 days after initial EVL, and intraprocedural adverse event rates between the minimal and maximal EVL groups.

\section{MATERIALS AND METHODS}

\section{Patients}

This was multicenter retrospective observational comparative study conducted for all patients who underwent any intervention for varix at Hallym University Medical Center, which consists of five hospitals between January 1, 2011, and December 31, 2017. Exclusion criteria were as follows: (1) patients who underwent only prophylactic
EVL; (2) patients who underwent other primary therapy for variceal bleeding, such as endoscopic injection sclerotherapy; (3) patients who underwent other rescue therapies, such as transjugular intrahepatic portosystemic shunt, splenorenal shunt therapy (balloon-occluded retrograde transvenous obliteration or coil-assisted retrograde transvenous obliteration); (4) patients who refused admission; and (5) patients with insufficient data on medical records.

\section{Endoscopic procedures}

Patients were managed according to the American Association for the Study of Liver Diseases practice guidelines in which early vasoactive agents (e.g., somatostatin or terlipressin) should be initiated at the same time with admission, and endoscopic therapy should be performed within 12 hours after initial presentation. For successful hemostasis, appropriate endoscopic therapy could be applied according to the bleeding source (on the preferential basis EVL if the bleeding focuses were esophageal or cardiac varices, and endoscopic sclerotherapy if the bleeding focuses were fundal gastric varices). Prophylactic antibiotics were administered to all patients with acute variceal bleeding from initial presentation to 7th day. After index EVL, patients underwent repeat EVL, scheduled 1 to 3 weeks until the varices became small or were eradicated. Since then, the recommended interval of surveillance endoscopy could be increased from every 1 or 2 months to every 4 or 5 months, and then sequentially maintained every 6 to 12 months indefinitely. ${ }^{13}$ All EVL procedures were performed by an experienced endoscopist who had performed approximately 1,000 cases of standard upper endoscopy and 20 cases of EVL per year. Furthermore, we did not apply a multiband device during emergent EVL for acute variceal bleeding.

\section{Definition and study endpoints}

Acute variceal bleeding was classified as an active bleeding (current oozing or spurting type bleeding on the varix) or the stigmata suggesting recent bleeding on the grade 2 (F2) or higher size of varices. ${ }^{14}$ Stigmata of recent bleeding was defined as the presence of a pin-point ulceration on the varix, adherent clot, or white protrusion in the setting of hematemesis but no other cause of upper gastrointestinal bleeding. ${ }^{15}$

Patients were divided into two groups, the minimal EVL group and the maximal EVL group, according to the extent of band ligation for treatment of acute variceal bleeding during the first endoscopic session. The minimal EVL group consisted of patients who underwent minimal band ligation targeting only current oozing or spurting type bleeding from the varix or stigmata (adherent clot, 
white protrusion, or pin-point ulceration) of a recent bleeding. The maximal EVL group included patients who underwent as many band ligations as possible for potential bleeding (positive red color sign or huge varices) as well as active variceal bleeding. Rebleeding was defined as variceal bleeding after initial bleeding control. ${ }^{16}$ If rebleeding occurred within 5 days from the initial EVL, the initial therapy was considered to have failed. ${ }^{16}$ Rebleeding events evaluated at other hospitals were included if the clinical and endoscopic information was exact and available.

Hypoxia was defined as desaturation (oxygen saturation $\left.\left[\mathrm{SpO}_{2}\right]<90 \%\right)$. Shock was defined as state of circulatory failure manifested by falling the systolic blood pressure (SBP) below $90 \mathrm{~mm}$ Hg. Furthermore, bradycardia was defined as a heart rate of less than 60 beats per minute.

The primary endpoint of the study was 5-day treatment failure, which was defined as failure to control variceal bleeding, early rebleeding, or death within 5 days. ${ }^{17}$ The secondary outcomes included 30-day rebleeding, 30-day mortality after initial EVL, and intraprocedural adverse event rates in both groups. We also evaluated clinical outcomes according to the subgroups of the Model for EndStage Liver Disease (MELD) score (MELD $\geq 15$ and MELD $<15$ subgroups).

\section{Statistical analysis}

Factors associated with treatment failure were assessed using the logistic regression analysis. The Kaplan-Meier plots and the log-rank tests were used to compare the overall survival between the maximal and minimal EVL groups. Additionally, the Cox proportional hazard model was used to identify the risk factors for 30-day mortality. In the multivariable logistic regression and Cox proportional hazard models, age, sex, and variables with p-value of less than 0.1 in the univariable logistic regression model were selected as covariates. In the analysis of rebleeding risk within 30 days, the Fine and Gray ${ }^{18}$ competing risk regression model was used because death is a competing risk for rebleeding. All reported p-values are two-sided, and $\mathrm{p}$-values $<0.05$ were considered statistically significant. All statistical analyses were conducted using $\mathrm{R}$ statistical software version 4.0.2 (R Foundation for Statistical Computing, Vienna, Austria).

\section{Ethical guidelines}

The Institutional Review Board of the Hallym University Medical Center approved this study (IRB number: 2017-07-005). In addition, this study was conducted in accordance with good clinical practice guidelines, the Declaration of Helsinki, and the Health Insurance Portability and Accountability Act. Since this was a retrospective analysis, the need for informed consent was waived.

\section{RESULTS}

\section{Study population and baseline characteristics}

A total of 1,823 patients underwent intervention for var-

Patients underwent endoscopic intervention for varix $(n=1,823)$

Excluded $(n=450)$

- Patients who underwent only prophylactic EVL $(n=257)$

- Patients who underwent primary EIS $(n=91)$

- Patients who underwent shunt therapy $(n=17)$

- Patients who underwent primary BRTO or CRTO $(n=24)$

- Patients who refused admission $(n=9)$

- Patients with insufficient medical record $(n=52)$

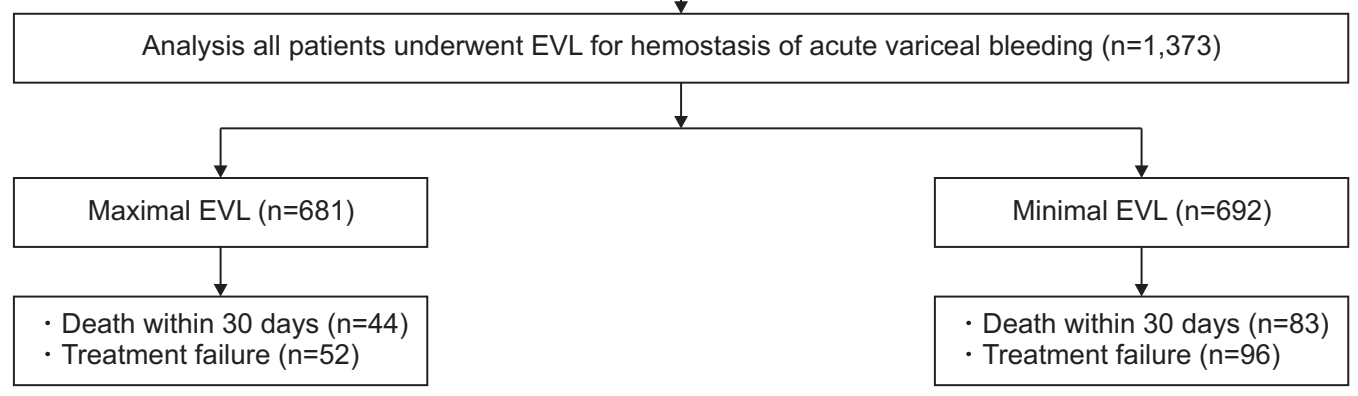

Fig. 1. Study flow diagram. Treatment failure was defined as the failure to control bleeding, early rebleeding, or death within 5 days.

EVL, endoscopic variceal ligation; EIS, endoscopic injection sclerotherapy; BRTO, balloon-occluded retrograde transvenous obliteration; CRTO, coil-assisted retrograde transvenous obliteration. 
ix at Hallym University Medical Centers during the study period; of these, 450 patients were excluded for the following reasons: underwent only prophylactic EVL $(n=257)$, primary endoscopic injection sclerotherapy $(n=91)$, transjugular intrahepatic portosystemic shunt $(n=17)$, or primary balloon-occluded retrograde transvenous obliteration or coil-assisted retrograde transvenous obliteration $(\mathrm{n}=24)$; refused admission $(\mathrm{n}=9)$; and had insufficient details on medical records $(n=52)$. The remaining 1,373 patients were included in the analyses and divided into the maximal EVL $(n=681)$ and minimal EVL groups $(n=692)$ according to the extent of band ligation during the first EVL session (Fig. 1).

Table 1 presents the baseline characteristics of the included patients. The mean age and proportion of males were 55.6 years and $79.9 \%$ in the maximal EVL group and 55.4 years and $81.6 \%$ in the minimal EVL group, respectively. Regarding the causes of liver cirrhosis, the maximal EVL group had a higher rate of hepatitis B virus infection, but there was no difference between the groups for other causes. The MELD score and Child-Turcotte-Pugh (CTP) score as parameters of liver function were significantly higher in the minimal EVL group than in the maximal EVL group (MELD: $11.2 \pm 5.1$ vs $10.3 \pm 4.1, \mathrm{p}<0.001$; CTP: $10.1 \pm 2.5$ vs $9.7 \pm 2.4, \mathrm{p}=0.008)$. The history of EVL did not differ between the groups (maximal vs minimal: $37.4 \%$ vs $37.3 \%, \mathrm{p}<0.956$ ). Additionally, there was no difference between groups regarding patients who were prescribed nonselective beta-blockers as prophylaxis for variceal bleeding (maximal vs minimal: $30.8 \%$ vs $31.1 \%, \mathrm{p}=0.953$ ).

\section{Endoscopic findings of varices}

Table 2 shows the endoscopic findings of the varices. The most common source of bleeding in each group was the esophageal varix. The red color sign and higher grades of varices were more common in the maximal EVL group than in the minimal EVL group (red color sign: $89.0 \%$ vs $83.1 \%$, $\mathrm{p}=0.002$; grade $\mathrm{F} 3: 43.5 \%$ vs $32.8 \%$, $\mathrm{p}<0.001$ ).

Table 1. Baseline Characteristics of Included Patients

\begin{tabular}{|c|c|c|c|}
\hline Variable & Maximal EVL ( $n=681)$ & Minimal EVL ( $n=692)$ & p-value \\
\hline Age, yr & $55.6 \pm 11.4$ & $55.4 \pm 11.4$ & 0.788 \\
\hline Male sex & $544(79.9)$ & $565(81.6)$ & 0.412 \\
\hline $\mathrm{BMI}, \mathrm{kg} / \mathrm{m}^{2}$ & $23.2 \pm 3.4$ & $22.9 \pm 3.3$ & 0.055 \\
\hline Current smoking & 285 (41.9) & 297 (42.9) & 0.702 \\
\hline Alcohol intake & $343(50.4)$ & $396(57.2)$ & 0.011 \\
\hline \multicolumn{4}{|l|}{ Etiology of liver disease } \\
\hline HBV & 228 (33.5) & $173(25.0)$ & 0.001 \\
\hline HCV & $49(7.2)$ & $43(6.2)$ & 0.518 \\
\hline Alcohol & $283(41.6)$ & 262 (37.9) & 0.168 \\
\hline Others & 109 (16.0) & $115(16.6)$ & 0.771 \\
\hline \multicolumn{4}{|l|}{ Initial laboratory findings } \\
\hline $\mathrm{WBC}, / \mu \mathrm{L}$ & $6,110(4,100-9,300)$ & $7,400(5,375-10,550)$ & $<0.001$ \\
\hline Hemoglobin, g/dL & $9.6(7.5-11.7)$ & $9.3(7.5-11.1)$ & 0.106 \\
\hline Platelet, $\times 10^{3} / \mu \mathrm{L}$ & $100(67-135)$ & 101 (70-137) & 0.555 \\
\hline AST, IU/L & $52(34-102)$ & $58(36-117)$ & 0.031 \\
\hline$A L T, I U / L$ & $31(20-50)$ & $29(19-48)$ & 0.183 \\
\hline Creatinine, mg/dL & $0.8(0.7-1.1)$ & $0.9(0.7-1.2)$ & 0.002 \\
\hline Albumin, g/dL & $3.2(2.8-3.5)$ & $3.0(2.6-3.4)$ & $<0.001$ \\
\hline Total bilirubin, mg/dL & $1.5(0.9-2.6)$ & $1.7(1.0-3.3)$ & 0.004 \\
\hline PT, INR & $1.4(1.2-1.6)$ & $1.4(1.3-1.7)$ & 0.005 \\
\hline MELD score & $10.3 \pm 4.1$ & $11.2 \pm 5.1$ & $<0.001$ \\
\hline CTP classification & & & 0.008 \\
\hline$A(5-6)$ & $14(2.1)$ & $14(2.0)$ & \\
\hline$B(7-9)$ & 383 (56.2) & $332(48.0)$ & \\
\hline$C(10-15)$ & $284(41.7)$ & $346(50.0)$ & \\
\hline Presence of $\mathrm{HCC}$ & 155 (22.8) & $149(21.5)$ & 0.603 \\
\hline History of HEP & $82(12.0)$ & 90 (13.0) & 0.625 \\
\hline History of EVL & $255(37.4)$ & 258 (37.3) & 0.956 \\
\hline Usage of nonselective beta blocker & $210(30.8)$ & $215(31.1)$ & 0.953 \\
\hline SBP at presentation, $\mathrm{mm} \mathrm{Hg}$ & $117.5 \pm 19.8$ & $116.8 \pm 22.6$ & 0.544 \\
\hline
\end{tabular}

Data are presented as mean \pm SD, number $(\%)$, or median (interquartile range).

EVL, endoscopic variceal ligation; BMI, body mass index; HBV, hepatitis B virus; HCV, hepatitis C virus; WBC, white blood cell; AST, aspartate aminotransferase; ALT, alanine aminotransferase; PT, prothrombin time; INR, International normalized ratio; MELD, Model for End-Stage Liver Disease; CTP, Child-Turcotte-Pugh; HCC, hepatocellular carcinoma; HEP, hepatic encephalopathy; SBP, systolic blood pressure. 
Table 2. Comparison of Endoscopic Findings between the Maximal and Minimal EVL Groups

\begin{tabular}{|c|c|c|c|}
\hline Variable & Maximal EVL (n=681) & Minimal EVL (n=692) & $\mathrm{p}$-value \\
\hline Bleeding source & & & 0.624 \\
\hline Esophageal varix & 562 (82.5) & 564 (81.5) & \\
\hline Gastric varix & 119 (17.5) & 128 (18.5) & \\
\hline Endoscopic classification & & & $<0.001$ \\
\hline Active variceal bleeding & $265(38.9)$ & $461(66.6)$ & \\
\hline High-risk stigmata & $416(61.1)$ & $231(33.4)$ & \\
\hline Grade of varix & & & $<0.001$ \\
\hline $\mathrm{F} 1$ & $37(5.4)$ & $101(14.6)$ & \\
\hline F2 & $348(51.1)$ & $364(52.6)$ & \\
\hline F3 & $296(43.5)$ & 227 (32.8) & \\
\hline Upper margin of varix & & & 0.080 \\
\hline Stomach cardia & $16(2.3)$ & $15(2.2)$ & \\
\hline Lower esophagus & $138(20.3)$ & 164 (23.7) & \\
\hline Mid esophagus & 387 (56.8) & 405 [58.5] & \\
\hline Upper esophagus & $140(20.6)$ & $108(15.6)$ & \\
\hline Color of varix & & & 0.418 \\
\hline Blue & 638 (93.7) & 656 (94.8) & \\
\hline White & 43 (6.3) & $36(5.2)$ & \\
\hline Red color sign & $606(89.0)$ & $575(83.1)$ & 0.002 \\
\hline Total procedure time, min & $15.1 \pm 9.4$ & $11.4 \pm 9.3$ & $<0.001$ \\
\hline
\end{tabular}

Data are presented as number (\%) or mean \pm SD.

$E V L$, endoscopic variceal ligation.

Table 3. Clinical Outcomes and Adverse Events between the Maximal and Minimal EVL Groups

\begin{tabular}{|c|c|c|c|}
\hline Variable & Maximal EVL (n=681) & Minimal EVL (n=692) & $\mathrm{p}$-value \\
\hline Numbers of band at first session & $4.8 \pm 2.0$ & $1.6 \pm 0.9$ & $<0.001$ \\
\hline Requiring blood transfusion within 72 hours & $398(58.4)$ & $552(79.8)$ & $<0.001$ \\
\hline Units of packed RBCs & $3.7 \pm 3.4$ & $3.9 \pm 2.8$ & 0.221 \\
\hline \multicolumn{4}{|l|}{ Adverse event during EVL } \\
\hline Aspiration pneumonia & $11(1.6)$ & $16(2.3)$ & 0.438 \\
\hline Hypoxia & $20(2.9)$ & $6(0.9)$ & 0.005 \\
\hline Bradycardia & $6(0.9)$ & $6(0.9)$ & $>0.999$ \\
\hline Shock & $23(3.4)$ & 9 (1.3) & 0.012 \\
\hline Death & $5(0.7)$ & $8(1.2)$ & 0.579 \\
\hline Treatment failure* & $52(7.6)$ & $96(13.9)$ & $<0.001$ \\
\hline Rebleeding within 30 days & $21(3.2)$ & $31(4.7)$ & 0.203 \\
\hline HEP after EVL within 30 days & $35(5.1)$ & $72(10.4)$ & $<0.001$ \\
\hline 30-Day mortality & $44(6.5)$ & $83(12.0)$ & $<0.001$ \\
\hline Cause of death & & & 0.009 \\
\hline Variceal bleeding & $9(1.3)$ & $14(2.0)$ & \\
\hline $\mathrm{HCC}$ & $3(0.4)$ & 9 (1.3) & \\
\hline Hepatic failure & $30(4.4)$ & $52(7.5)$ & \\
\hline Infection & $1(0.1)$ & $2(0.3)$ & \\
\hline Others & $1(0.1)$ & $6(0.9)$ & \\
\hline Duration of follow-up, day & $770(225-1,466)$ & $714(116-1,549)$ & 0.186 \\
\hline Prophylactic EVL on same hospitalization & $184(27.0)$ & 324 (46.8) & $<0.001$ \\
\hline Number of endoscopic sessions for complete eradication & $1.4 \pm 0.6$ & $1.5 \pm 0.6$ & 0.001 \\
\hline 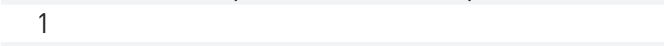 & 441 (64.8) & $380(54.9)$ & \\
\hline 2 & 228 (33.5) & $297(42.9)$ & \\
\hline 3 & $9(1.3)$ & $11(1.6)$ & \\
\hline 4 & $1(0.1)$ & $1(0.1)$ & \\
\hline 5 & 0 & $3(0.4)$ & \\
\hline 6 & $2(0.3)$ & 0 & \\
\hline Length of hospital stay, day & $9(6-14)$ & $9(7-14)$ & 0.267 \\
\hline
\end{tabular}

Data are presented as mean \pm SD, number (\%), or median (interquartile range).

EVL, endoscopic variceal ligation; RBC, red blood cell; HEP, hepatic encephalopathy; HCC, hepatocellular carcinoma.

*Treatment failure was defined as failure to control bleeding, early rebleeding, or death within 5 days. 
Furthermore, 66.6\% (461/692) of patients in the minimal EVL group and 38.9\% (265/681) of patients in the maximal EVL group had active variceal bleeding $(\mathrm{p}<0.001)$. The total procedure time was shorter in the minimal EVL group than in the maximal EVL group (11.4 \pm 9.3 minutes vs $15.1 \pm 9.4$ minutes, $\mathrm{p}<0.001$ ).

\section{Clinical outcomes and adverse events of EVL}

Clinical outcomes and adverse events associated with EVL according to the extent of EVL are shown in Table 3. The number of bands used during the first endoscopic session in the maximal EVL group was significantly higher than that in the minimal EVL group ( $4.8 \pm 2.0$ vs $1.6 \pm 0.9$, $\mathrm{p}<0.001)$. Within 72 hours after EVL, red blood cell transfusion was required more often in the minimal EVL group than in the maximal EVL group ( $79.8 \%$ vs $58.4 \%$, $\mathrm{p}<0.001)$, without significant differences in the total units of transfused red blood cells between groups.

Regarding intraprocedural adverse events, the minimal EVL group presented significantly lower rates of hypoxia $(0.9 \%$ vs $2.9 \%, \mathrm{p}=0.005)$ and shock $(1.3 \%$ vs $3.4 \%$, $\mathrm{p}=0.012)$ than the maximal EVL group. Treatment failure

Table 4. Factors Associated with Treatment Failure

\begin{tabular}{|c|c|c|c|c|c|c|}
\hline \multirow{2}{*}{ Variable } & \multirow{2}{*}{ No. } & \multirow{2}{*}{ Treatment failure, No. (\%)* } & \multicolumn{2}{|c|}{ Univariable analysis } & \multicolumn{2}{|c|}{ Multivariable analysis } \\
\hline & & & OR $(95 \% \mathrm{Cl})$ & $\mathrm{p}$-value & OR $(95 \% \mathrm{Cl})$ & $\mathrm{p}$-value \\
\hline \multicolumn{7}{|l|}{ Extent of EVL } \\
\hline Maximal EVL & 681 & $52(7.6)$ & 1 & & 1 & \\
\hline Minimal EVL & 692 & 96 (13.9) & $1.95(1.37-2.78)$ & $<0.001$ & $1.60(1.06-2.41)$ & 0.024 \\
\hline \multicolumn{7}{|l|}{ Age } \\
\hline$<60 \mathrm{yr}$ & 926 & $91(9.8)$ & 1 & & 1 & \\
\hline$\geq 60 \mathrm{yr}$ & 447 & $57(12.8)$ & $1.34(0.94-1.91)$ & 0.102 & $1.54(1.03-2.31)$ & 0.036 \\
\hline \multicolumn{7}{|l|}{ Sex } \\
\hline Male & 1,109 & $118(10.6)$ & $0.93(0.61-1.42)$ & 0.733 & & \\
\hline Female & 264 & $30(11.4)$ & 1 & & & \\
\hline \multicolumn{7}{|l|}{ BMI } \\
\hline$<25 \mathrm{~kg} / \mathrm{m}^{2}$ & 1,029 & $114(11.1)$ & 1 & & & \\
\hline$\geq 25 \mathrm{~kg} / \mathrm{m}^{2}$ & 344 & 34 (9.9) & $0.88(0.59-1.32)$ & 0.536 & & \\
\hline \multicolumn{7}{|l|}{ Current smoking } \\
\hline Absent & 791 & $87(11.0)$ & 1 & & & \\
\hline Present & 582 & $61(10.5)$ & $0.95(0.67-1.34)$ & 0.760 & & \\
\hline \multicolumn{7}{|l|}{ Alcohol consumption } \\
\hline Absence & 634 & $72(11.4)$ & 1 & & & \\
\hline Presence & 739 & $76(10.3)$ & $0.89(0.64-1.26)$ & 0.523 & & \\
\hline \multicolumn{7}{|l|}{ MELD } \\
\hline$<15$ & 1,178 & $68(5.8)$ & 1 & & 1 & \\
\hline$\geq 15$ & 195 & $80(41.0)$ & $11.36(7.80-16.54)$ & $<0.001$ & $9.31(6.17-14.04)$ & $<0.001$ \\
\hline \multicolumn{7}{|l|}{ CTP } \\
\hline$A$ or $B$ & 743 & $45(6.1)$ & 1 & & 1 & \\
\hline C & 630 & 103 (16.3) & $3.03(2.10-4.38)$ & $<0.001$ & $1.60(1.05-2.43)$ & 0.029 \\
\hline \multicolumn{7}{|l|}{$\mathrm{HCC}$} \\
\hline Absent & 1,069 & $100(9.4)$ & 1 & & 1 & \\
\hline Present & 304 & $48(15.8)$ & $1.82(1.25-2.63)$ & 0.002 & $1.80(1.18-2.75)$ & 0.006 \\
\hline \multicolumn{7}{|l|}{ SBP at presentation } \\
\hline$\geq 90 \mathrm{~mm} \mathrm{Hg}$ & 1,286 & 119 (9.3) & 1 & & 1 & \\
\hline$<90 \mathrm{~mm} \mathrm{Hg}$ & 87 & 29 (33.3) & $4.90(3.02-7.96)$ & $<0.001$ & $3.82(2.17-6.73)$ & $<0.001$ \\
\hline \multicolumn{7}{|l|}{ Grade of varix } \\
\hline F1 & 138 & $16(11.6)$ & 1 & & & \\
\hline $\mathrm{F} 2$ or $\mathrm{F} 3$ & 1,235 & $132(10.7)$ & $0.91(0.53-1.58)$ & 0.745 & & \\
\hline \multicolumn{7}{|l|}{ Red color sign } \\
\hline Absent & 192 & 19 (9.9) & 1 & & & \\
\hline Present & 1,181 & $129(10.9)$ & $1.12(0.67-1.86)$ & 0.670 & & \\
\hline \multicolumn{7}{|l|}{ Endoscopic classification } \\
\hline Active variceal bleeding & 726 & 90 (12.4) & $1.44(1.01-2.04)$ & 0.041 & $1.12(0.75-1.68)$ & 0.574 \\
\hline High-risk stigmata & 647 & $58(9.0)$ & 1 & & 1 & \\
\hline
\end{tabular}

OR, odds ratio; $\mathrm{Cl}$, confidence interval; EVL, endoscopic variceal ligation; BMI, body mass index; MELD, Model for End-Stage Liver Disease; CTP, Child-Turcotte-Pugh; HCC, hepatocellular carcinoma; SBP, systolic blood pressure.

*Treatment failure was defined as failure to control bleeding, early rebleeding, or death within 5 days. 


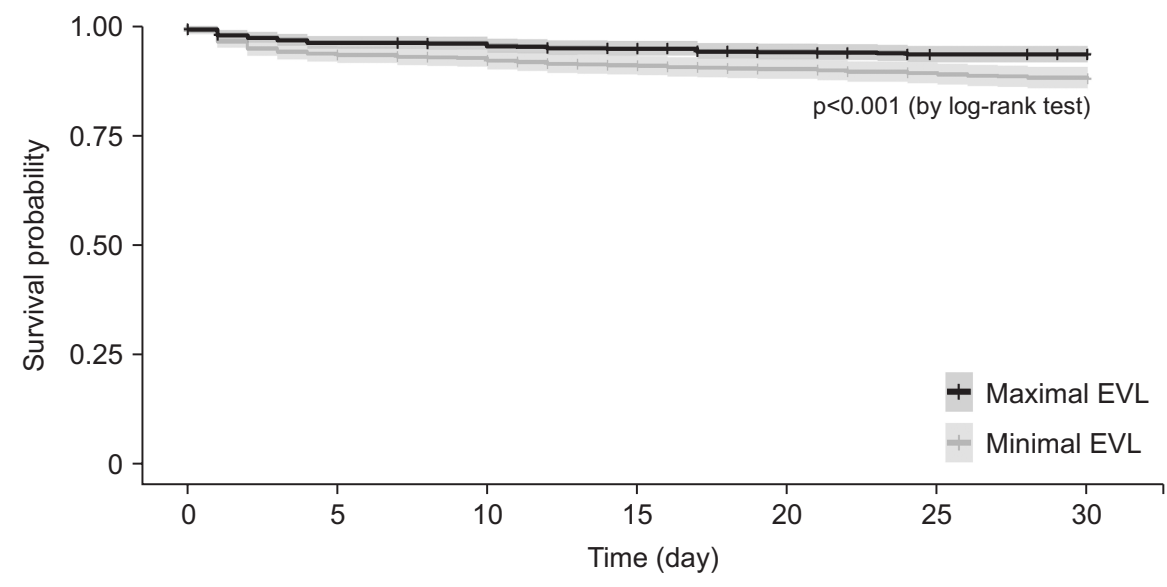

Number at risk

$\begin{array}{llllllll}\text { Maximal EVL } & 681 & 652 & 649 & 634 & 624 & 617 & 615\end{array}$

Fig. 2. Kaplan-Meier plots for overall

$\begin{array}{llllllll}\text { Minimal EVL } & 692 & 647 & 637 & 613 & 599 & 587 & 580\end{array}$

survival within 30 days.

$E V L$, endoscopic variceal ligation.

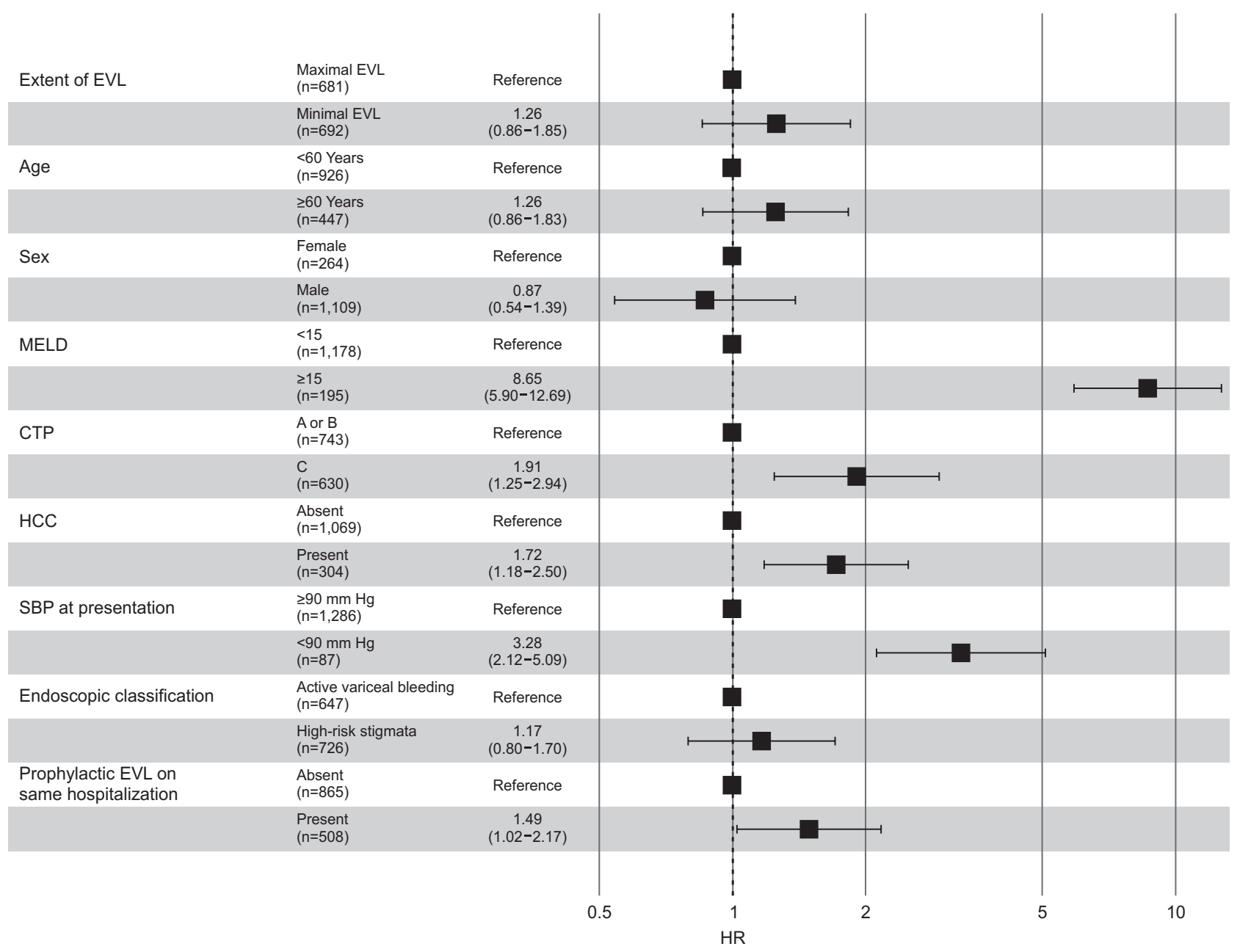

Fig. 3. Forest plot of the Cox proportional hazard model for 30-day mortality. The black rectangle and bar indicate the HR and $95 \%$ confidence interval, respectively.

EVL, endoscopic variceal ligation; MELD, Model for End-Stage Liver Disease; CTP, Child-Turcotte-Pugh; HCC, hepatocellular carcinoma; SBP, systolic blood pressure; HR, hazard ratio. 
rates were higher in the minimal EVL group than in the maximal EVL group ( $13.9 \%$ vs $7.6 \%, p<0.001)$. However, the rebleeding rate within 30 days after EVL did not differ between the groups. A total of 127 patients in the entire cohort died within the first 30 days. The most common cause of death was hepatic failure in both groups.

Supplementary Table 1 shows the clinical outcomes of the subgroup analyses according to the baseline MELD score. Although the treatment failure rate was higher in the MELD $\geq 15$ subgroup than in the MELD $<15$ subgroup, it did not differ between the minimal and maximal EVL groups in the MELD $\geq 15$ subgroup. Hypoxia and shock also did not differ between the minimal and maximal EVL groups in the MELD $\geq 15$ subgroup. In the MELD $<15$ subgroup, however, the treatment failure rate in the minimal EVL group was higher than that in the maximal EVL group.

The multivariable logistic regression model for treatment failure is presented in Table 4. After adjusting for potential confounding variables, minimal EVL was shown to be associated with treatment failure (odds ratio, 1.60; $95 \%$ confidence interval [CI], 1.06 to 2.41 ). Age $\geq 60$ years, MELD score $\geq 15$, CTP classification C, presence of hepatocellular carcinoma (HCC), and SBP $<90 \mathrm{~mm} \mathrm{Hg}$ on initial presentation were also risk factors for treatment failure.

\section{Survival analysis}

Supplementary Table 2 shows the competing risk analysis for 30-day rebleeding. MELD score $\geq 15$, presence of HCC, and prophylactic EVL during the same hospitalization were associated with rebleeding (MELD $\geq 15$ : subdistribution hazard ratio [SHR], 3.09; 95\% CI, 1.70 to 5.61; HCC: SHR, 2.23; 95\% CI, 1.27 to 3.94; prophylactic EVL: SHR, 1.78; 95\% CI, 1.02 to 3.10). However, minimal EVL did not affect the 30-day rebleeding (SHR, 0.84; 95\% CI, 0.47 to 1.49 ).

Kaplan-Meier plots showed that the maximal EVL group had superior overall survival within 30 days when compared with the minimal EVL group ( $p<0.001)$ (Fig. 2). In the MELD $\geq 15$ subgroup, no significant difference in 30-day mortality was identified between the minimal and maximal EVL groups, whereas the maximal EVL group was superior to the minimal EVL group in the MELD $<15$ subgroup (Supplementary Fig. 1). However, the significant impact of minimal EVL on the 30-day mortality was not identified in the Cox proportional hazard model after adjustment for potential confounding variables (Fig. 3). MELD score $\geq 15, \mathrm{SBP}<90 \mathrm{~mm} \mathrm{Hg}$ at initial presentation, CTP classification C, presence of HCC, and prophylactic EVL during the same hospitalization were significant risk factors for 30-day mortality (MELD $\geq 15$ : hazard ratio [HR],
8.65; $95 \%$ CI, 5.90 to 12.69 ; SBP <90 mm Hg: HR, 3.28; 95\% CI, 2.12 to 5.09; CTP classification C: HR, 1.91; 95\% CI, 1.25 to 2.94; presence of HCC: HR, 1.72; 95\% CI, 1.18 to 2.50; prophylactic EVL: HR, 1.49; 95\% CI, 1.02 to 2.17).

\section{DISCUSSION}

The current study reported the outcomes of patients with acute variceal bleeding according to the extent of EVL that they received at the initial presentation. The overall treatment success rate, rebleeding rate, and mortality rate in our study were consistent with the results reported in previous studies. ${ }^{12,19-21}$ In our study, a minimal extent of EVL increased the risk of treatment failure. However, the 30-day rebleeding rate after initial EVL did not differ between the groups, and the rates of hypoxia and shock were lower in the minimal EVL group than in the maximal EVL group.

EVL is a standard therapy for esophageal and cardiac varices; however, only a few studies are available on the optimal number of bands that should be used during the procedure. Harewood et al..$^{22}$ compared the number of bands per session and the bleeding incidence. In their study, which had a small sample size of 40 patients, they could not find a significant correlation between the median number of bands used for EVL and bleeding episodes for rebleeding and non-rebleeding groups. Another study reported a correlation between the number of ligation bands and bleeding from ligation ulcers; patients with bleeding at ligation sites were treated with significantly more ligation bands. ${ }^{15}$ Therefore, they assumed that the application of more bands could be a risk factor for additional bleeding from ulcers. Furthermore, Ramirez et al. ${ }^{12}$ conducted a prospective study involving 86 patients that compared the placement of a maximum of six bands per session with the placement of more than six bands per session. According to the results of that study, the placement of more than six bands per session was not associated with better outcomes in terms of variceal bleeding recurrence and overall mortality. Although that study provided some indication of the optimal number of bands for EVL, the cutoff number of bands was decided based on the multiband device containing six bands that was available on the market at that time.

Data regarding differences in clinical outcomes when using fewer than six bands are lacking. During our study, the minimal EVL group received only 1.6 bands per session, whereas the maximal EVL group received 4.8 bands. The high treatment failure rate of the minimal EVL group suggests that the minimal use of bands is not an optimal strategy for hemostasis of acute variceal bleeding. How- 
ever, maximal EVL does not guarantee less rebleeding and better survival. Given that hypoxia and shock were more common in the maximal EVL group than in the minimal EVL group, the minimal EVL strategy may be chosen for patients with variceal bleeding with poor general conditions or hemodynamic instability. Although the effects of second-look endoscopic treatment were not analyzed during this study, Wang et al. ${ }^{23}$ recently reported that secondlook endoscopic treatment, which is performed after EVL for acute bleeding of large endoscopic varices, is an option for reducing early rebleeding.

In addition to the EVL extent, high MELD score, advanced grade of CTP classification, presence of HCC, and shock at presentation were also significantly associated with treatment failure in our study. Hemodynamic instability and baseline patient performance are well-known conditions related to morbidity and mortality rates of acute variceal bleeding. $8,24,25$ These factors are also related to the 30-day mortality of patients. However, the extent of EVL was not associated with 30-day mortality. In the subgroup analysis, according to the MELD score, there was no significant difference in terms of treatment failure, rebleeding, and mortality between the minimal and maximal EVL groups in patients with MELD $\geq 15$. In other words, the minimal EVL strategy may be a feasible option for patients with acute variceal bleeding with a high MELD score.

The possibility that the patient's underlying condition influenced the selection of the EVL method should be considered when interpreting our results. As can be seen from Table 2, the baseline endoscopic findings of the presence of active bleeding, the red color sign, and the grades of the varices differed between the groups. The presence of active bleeding may be associated with low baseline SBP at presentation and a higher requirement for transfusion, leading physicians to use the minimal number of EVL bands for short procedures. Additionally, the higher incidence of high-risk stigmata, the red color sign, and F3 grade varices might indicate the need for a greater number of EVL bands.

Although this was a multicenter, large-scale study, it had several limitations. First, the extent of EVL was determined at the clinicians' discretion after considering the patient's general condition because clinical consensus regarding the extent of EVL has not been established yet. It means that the extent of EVL is a mediator rather than an exposure in this study. Although we adjusted for potentially confounding variables, including MELD score, CTP classification, presence of HCC, endoscopic classification (active variceal bleeding vs high-risk stigmata), and shock, hidden or unmeasured factors may remain. We hope to this limitation can be overcome through randomized controlled trials.
Second, patients in both groups were not evenly matched, especially in terms of baseline liver function, CTP class, and MELD score. However, our study involved the largest number of patients with acute variceal bleeding, and we tried to compensate for the selection bias as much as possible with sufficient statistical power. Third, the EVL protocols were not uniform across patients because of the retrospective nature of the study. A prospective study with predefined EVL protocols may help to reach a definitive conclusion.

Despite these limitations, our multicenter real-world data provide a better understanding of the optimal extent of EVL for patients with acute variceal bleeding. Maximal EVL may be a better treatment option for acute variceal bleeding because of the lower risk of treatment failure than minimal EVL. However, the maximal EVL was associated with more frequent hypoxia and shock during EVL compared to minimal EVL. Additionally, 30-day rebleeding and mortality did not differ between the minimal and maximal EVL groups. Taken together, the minimal EVL strategy may be considered in selective patients with poor general conditions or hemodynamic instability.

\section{CONFLICTS OF INTEREST}

No potential conflict of interest relevant to this article was reported.

\section{AUTHOR CONTRIBUTIONS}

Study concept and design: J.H. Jung, J.H. Jo, C.H.P., S.W.P. Acquisition of data: J.H. Jung, S.E.K., C.S.B., S.I.S., S.W.P. Analysis and interpretation of data: J.H. Jung, J.H. Jo, C.H.P., S.W.P. Statistical analysis: C.H.P., S.W.P. Study supervision: C.H.P., S.W.P. Writing - original draft: J.H.J., J.H.J., C.H.P., S.W.P. Critical revision of the manuscript for important intellectual content: J.H. Jung, C.H.P.

\section{ORCID}

Jang Han Jung Jung Hyun Jo Sung Eun Kim Chang Seok Bang Seung In Seo Chan Hyuk Park Se Woo Park https://orcid.org/0000-0001-7875-2868 https://orcid.org/0000-0002-2641-8873 https://orcid.org/0000-0001-6236-780X https://orcid.org/0000-0003-4908-5431 https://orcid.org/0000-0003-4417-0135 https://orcid.org/0000-0003-3824-3481 https://orcid.org/0000-0003-1603-7468 


\section{SUPPLEMENTARY MATERIALS}

Supplementary materials can be accessed at https://doi. org/10.5009/gnl20375.

\section{REFERENCES}

1. Lay CS, Tsai YT, Teg CY, et al. Endoscopic variceal ligation in prophylaxis of first variceal bleeding in cirrhotic patients with high-risk esophageal varices. Hepatology 1997;25:1346-1350.

2. Lo GH, Lai KH, Cheng JS, Lin CK, Hsu PI, Chiang HT. Prophylactic banding ligation of high-risk esophageal varices in patients with cirrhosis: a prospective, randomized trial. J Hepatol 1999;31:451-456.

3. Djurdjevic D, Janosevic S, Dapcevic B, et al. Combined ligation and sclerotherapy versus ligation alone for eradication of bleeding esophageal varices: a randomized and prospective trial. Endoscopy 1999;31:286-290.

4. Pomier-Layrargues G, Villeneuve JP, Deschênes M, et al. Transjugular intrahepatic portosystemic shunt (TIPS) versus endoscopic variceal ligation in the prevention of variceal rebleeding in patients with cirrhosis: a randomised trial. Gut 2001;48:390-396.

5. Singh P, Pooran N, Indaram A, Bank S. Combined ligation and sclerotherapy versus ligation alone for secondary prophylaxis of esophageal variceal bleeding: a meta-analysis. Am J Gastroenterol 2002;97:623-629.

6. Garcia-Tsao G, Abraldes JG, Berzigotti A, Bosch J. Portal hypertensive bleeding in cirrhosis: risk stratification, diagnosis, and management. 2016 Practice guidance by the American Association for the Study of Liver Diseases. Hepatology 2017;65:310-335.

7. Saeed ZA, Stiegmann GV, Ramirez FC, et al. Endoscopic variceal ligation is superior to combined ligation and sclerotherapy for esophageal varices: a multicenter prospective randomized trial. Hepatology 1997;25:71-74.

8. Garcia-Tsao G, Sanyal AJ, Grace ND, Carey W; Practice Guidelines Committee of the American Association for the Study of Liver Diseases; Practice Parameters Committee of the American College of Gastroenterology. Prevention and management of gastroesophageal varices and variceal hemorrhage in cirrhosis. Hepatology 2007;46:922-938.

9. Seo YS. Prevention and management of gastroesophageal varices. Clin Mol Hepatol 2018;24:20-42.

10. Mao W, Wei XQ, Tao J, Zhen FP, Wen ZF, Wu B. The safety of combined sedation with propofol plus fentanyl for endoscopy screening and endoscopic variceal ligation in cirrhotic patients. J Dig Dis 2014;15:124-130.

11. Johanson JF, Rimm A. Optimal nonsurgical treatment of hemorrhoids: a comparative analysis of infrared coagulation, rubber band ligation, and injection sclerotherapy. Am J Gastroenterol 1992;87:1600-1606.

12. Ramirez FC, Colon VJ, Landan D, Grade AJ, Evanich E. The effects of the number of rubber bands placed at each endoscopic session upon variceal outcomes: a prospective, randomized study. Am J Gastroenterol 2007;102:1372-1376.

13. Al Traif I, Fachartz FS, Al Jumah A, et al. Randomized trial of ligation versus combined ligation and sclerotherapy for bleeding esophageal varices. Gastrointest Endosc 1999;50:1-6.

14. Kim SJ, Choi CW, Kang DH, et al. Emergency endoscopic variceal ligation in cirrhotic patients with blood clots in the stomach but no active bleeding or stigmata increases the risk of rebleeding. Clin Mol Hepatol 2016;22:466-476.

15. Petrasch F, Grothaus J, Mössner J, Schiefke I, Hoffmeister A. Differences in bleeding behavior after endoscopic band ligation: a retrospective analysis. BMC Gastroenterol 2010;10:5.

16. Albillos A, Zamora J, Martínez J, et al. Stratifying risk in the prevention of recurrent variceal hemorrhage: results of an individual patient meta-analysis. Hepatology 2017;66:1219-1231.

17. de Franchis R; Baveno V Faculty. Revising consensus in portal hypertension: report of the Baveno $\mathrm{V}$ consensus workshop on methodology of diagnosis and therapy in portal hypertension. J Hepatol 2010;53:762-768.

18. Fine JP, Gray RJ. A proportional hazards model for the subdistribution of a competing risk. J Am Stat Assoc 1999;94:496-509.

19. Ibrahim M, El-Mikkawy A, Abdel Hamid M, et al. Early application of haemostatic powder added to standard management for oesophagogastric variceal bleeding: a randomised trial. Gut 2019;68:844-853.

20. de la Peña J, Rivero M, Sanchez E, Fábrega E, Crespo J, PonsRomero F. Variceal ligation compared with endoscopic sclerotherapy for variceal hemorrhage: prospective randomized trial. Gastrointest Endosc 1999;49(4 Pt 1):417-423.

21. Sarin SK, Govil A, Jain AK, et al. Prospective randomized trial of endoscopic sclerotherapy versus variceal band ligation for esophageal varices: influence on gastropathy, gastric varices and variceal recurrence. J Hepatol 1997;26:826-832.

22. Harewood GC, Baron TH, Song LM. Factors predicting success of endoscopic variceal ligation for secondary prophylaxis of esophageal variceal bleeding. J Gastroenterol Hepatol 2006;21(1 Pt 2):237-241.

23. Wang AJ, Wang J, Zheng XL, et al. Second-look endoscopyguided therapy under sedation prevents early rebleeding after variceal ligation for acute variceal bleeding. J Dig Dis 2020;21:170-178.

24. Garcia-Tsao G, Bosch J. Management of varices and variceal hemorrhage in cirrhosis. N Engl J Med 2010;362:823-832.

25. Corley DA, Cello JP, Adkisson W, Ko WF, Kerlikowske K. Octreotide for acute esophageal variceal bleeding: a metaanalysis. Gastroenterology 2001;120:946-954. 